\title{
ASYMPTOTIC SHARPNESS \\ OF A BERNSTEIN-TYPE INEQUALITY \\ FOR RATIONAL FUNCTIONS IN $H^{2}$
}

\author{
R. ZAROUF
}

\begin{abstract}
A Bernstein-type inequality for the standard Hardy space $H^{2}$ in the unit disk $\mathbb{D}=\{z \in \mathbb{C}:|z|<1\}$ is considered for rational functions in $\mathbb{D}$ having at most $n$ poles all outside of $\frac{1}{r} \mathbb{D}, 0<r<1$. The asymptotic sharpness is shown as $n \rightarrow \infty$ and $r \rightarrow 1$.
\end{abstract}

\section{§1. INTRODUCTION}

First, we recall the classical Bernstein inequality for polynomials: let $\mathcal{P}_{n}$ denote the class of all polynomials $P=\sum_{k=0}^{n} a_{k} z^{k}$ with complex coefficients, of degree $n$. Let

$$
\|P\|_{2}=\frac{1}{\sqrt{2 \pi}}\left(\int_{\mathbb{T}}|P(\zeta)|^{2} d \zeta\right)^{\frac{1}{2}}=\left(\sum_{k=0}^{n}\left|a_{k}\right|^{2}\right)^{\frac{1}{2}} .
$$

The classical inequality

$$
\left\|P^{\prime}\right\|_{2} \leq n\|P\|_{2}
$$

is known as Bernstein's inequality. A great number of refinements and generalizations of (1) have been obtained. See [RaSc, Part III] for an extensive study of that subject. The constant $n$ in (1) is obviously sharp (take $P=z^{n}$ ).

Now, let $\sigma=\left\{\lambda_{1}, \ldots, \lambda_{n}\right\}$ be a sequence in the unit disk $\mathbb{D}$; consider the finite Blaschke product $B_{\sigma}=\Pi_{i=1}^{n} b_{\lambda_{i}}$, where $b_{\lambda}=\frac{\lambda-z}{1-\bar{\lambda} z}$ is an elementary Blaschke factor for $\lambda \in \mathbb{D}$. Also, let $K_{B_{\sigma}}$ be the $n$-dimensional space defined by

$$
K_{B_{\sigma}}=\operatorname{Lin}\left(k_{\lambda_{i}}: i=1, \ldots, n\right),
$$

where $\sigma$ is a family of distinct elements of $\mathbb{D}$, and $k_{\lambda}=\frac{1}{1-\bar{\lambda} z}$ is the Szegö kernel associated with $\lambda$. An obvious modification allows us to generalize the definition of $K_{B_{\sigma}}$ to the case where the sequence $\sigma$ admits multiplicities.

Notice that in terms of the scalar product $(\cdot, \cdot)_{H^{2}}$ on $H^{2}$, an equivalent description of this space looks like this:

$$
K_{B_{\sigma}}=\left(B_{\sigma} H^{2}\right)^{\perp}=H^{2} \ominus B_{\sigma} H^{2},
$$

where $H^{2}$ stands for the standard Hardy space of the unit disk $\mathbb{D}$,

$$
H^{2}=\left\{f=\sum_{k \geq 0} \hat{f}(k) z^{k}: \sup _{0 \leq r<1} \int_{\mathbb{T}}|f(r z)|^{2} d m(z)<\infty\right\},
$$

$m$ being the normalized Lebesgue measure on $\mathbb{T}$. We notice that the case where $\lambda_{1}=$ $\lambda_{2}=\cdots=\lambda_{n}=0$ gives $K_{B_{\sigma}}=\mathcal{P}_{n}$. Our goal in this paper is to generalize the classical Bernstein inequality (1) to the spaces $K_{B_{\sigma}}$. Observe that every rational function with

2010 Mathematics Subject Classification. Primary 30H10, 30J10.

Key words and phrases. Bernstein inequality, finite Blaschke product, Hardy space. 
poles outside of $\overline{\mathbb{D}}$ lies in a space $K_{B_{\sigma}}$. It was already proved in [Z1] that if $r=\max _{j}\left|\lambda_{j}\right|$, and $f \in K_{B_{\sigma}}$, then

$$
\left\|f^{\prime}\right\|_{H^{2}} \leq \frac{5}{2} \frac{n}{1-r}\|f\|_{H^{2}}
$$

In fact, inequality $(2)$ is a partial case $(p=2)$ of the following Dyakonov's result Dya1 (which, in its turn, is a generalization of Levin's inequality $[\mathrm{L}$. corresponding to $p=\infty$ ): for every $p, 1<p \leq \infty$ there exists a constant $c_{p}>0$ such that

$$
\left\|f^{\prime}\right\|_{H^{p}} \leq c_{p}\left\|B^{\prime}\right\|_{\infty}\|f\|_{H^{p}}
$$

for all $f \in K_{B}$, where $B$ is a finite Blaschke product (of order $n$ ) and $\|\cdot\|_{\infty}$ means the norm in $L^{\infty}(\mathbb{T})$. For our partial case, our proof (in [Z1]) is different and the constant is slightly better. We note that, in general, Bernstein type inequalities have already been the subject of a lot of papers. Among others, Chapter 7 of the book $[\mathrm{BoEr}$ by P. Borwein and T. Erdélyi was devoted to such inequalities. This is also the case for Baranov's papers [B1, B2] and [B3], and also for the book DeLo] by R. A. DeVore and G. G. Lorentz.

Now a natural question arises: is the constant $\frac{5}{2} \frac{n}{1-r}$ in (2) sharp? For $r=0$ (the classical Bernstein case), we know that this is not the case, because constant $n$ is sharp. Below we show that the growth order $\frac{n}{1-r}$ as $n \rightarrow \infty$ and $r \rightarrow 1$ is sharp and give loose bounds for the numerical constants that arise.

\section{$\S 2$. The Result}

Theorem. Let $n \geq 1$, let $\sigma=\left\{\lambda_{1}, \ldots, \lambda_{n}\right\}$ be a sequence in the unit disk $\mathbb{D}$, and let $B_{\sigma}$ be the finite Blaschke product $B_{\sigma}=\Pi_{i=1}^{n} b_{\lambda_{i}}$, where $b_{\lambda}=\frac{\lambda-z}{1-\lambda z}$ is an elementary Blaschke factor for $\lambda \in \mathbb{D}$. Let $K_{B_{\sigma}}$ be the $n$-dimensional subspace of $H^{2}$ defined by

$$
K_{B_{\sigma}}=\left(B_{\sigma} H^{2}\right)^{\perp}=H^{2} \ominus B_{\sigma} H^{2} .
$$

Denote by $D$ the operator of differentiation on $\left(K_{B_{\sigma}},\|\cdot\|_{2}\right)$ :

$$
\begin{aligned}
D:\left(K_{B_{\sigma}},\|\cdot\|_{2}\right) & \rightarrow\left(H^{2},\|\cdot\|_{2}\right) \\
f & \mapsto f^{\prime},
\end{aligned}
$$

where $\|f\|_{2}=\|f\|_{H^{2}}=\frac{1}{\sqrt{2 \pi}}\left(\int_{\mathbb{T}}|f(\zeta)|^{2} d \zeta\right)^{\frac{1}{2}}$. For $r \in[0,1)$ and $n \geq 1$, we set

$$
C_{n, r}=\sup \left\{\|D\|_{K_{B_{\sigma}} \rightarrow H^{2}}: 1 \leq \# \sigma \leq n,|\lambda| \leq r \quad \text { for all } \lambda \in \sigma\right\} .
$$

(i) If $n=1$ and $\sigma=\{\lambda\}$, we have

$$
\|D\|_{K_{B_{\sigma}} \rightarrow H^{2}}=|\lambda|\left(\frac{1}{1-|\lambda|^{2}}\right)^{\frac{1}{2}} .
$$

If $n \geq 2$, then

where

$$
a(n, r) \frac{n}{1-r} \leq C_{n, r} \leq A(n, r) \frac{n}{1-r}
$$

$$
a(n, r) \geq \frac{1}{1+r}\left(1+5 r^{4}-\frac{4 r^{4}}{n}-\min \left(\frac{3}{4}, \frac{2}{n}\right)\right)^{\frac{1}{2}}
$$

and

(ii) Moreover, the sequence

$$
A(n, r) \leq 1+r+\frac{1}{\sqrt{n}}
$$

$$
\left(\frac{1}{n} C_{n, r}\right)_{n \geq 1}
$$


is convergent, and

for all $r \in[0,1)$.

$$
\lim _{n \rightarrow \infty} \frac{1}{n} C_{n, r}=\frac{1+r}{1-r}
$$

Proof of (i). The case where $n=1$. In this case, $K_{B}=\mathbb{C} e_{1}$, where

$$
e_{1}=\frac{\left(1-|\lambda|^{2}\right)^{\frac{1}{2}}}{(1-\bar{\lambda} z)}, \quad|\lambda| \leq r
$$

( $e_{1}$ being of norm 1 in $\left.H^{2}\right)$. Calculating

$$
e_{1}^{\prime}=\frac{\bar{\lambda}\left(1-|\lambda|^{2}\right)^{\frac{1}{2}}}{(1-\bar{\lambda} z)^{2}}
$$

and

$$
\begin{aligned}
\left\|e_{1}^{\prime}\right\|_{H^{2}} & =|\lambda|\left(1-|\lambda|^{2}\right)^{\frac{1}{2}}\left\|\frac{1}{(1-\bar{\lambda} z)^{2}}\right\|_{H^{2}}=|\lambda|\left(1-|\lambda|^{2}\right)^{\frac{1}{2}}\left(\sum_{k \geq 0}(k+1)|\lambda|^{2 k}\right)^{\frac{1}{2}} \\
& =|\lambda|\left(1-|\lambda|^{2}\right)^{\frac{1}{2}} \frac{1}{\left(1-|\lambda|^{2}\right)}=|\lambda|\left(\frac{1}{1-|\lambda|^{2}}\right)^{\frac{1}{2}},
\end{aligned}
$$

we get

$$
\left\|D_{\mid K_{B_{\sigma}}}\right\|=|\lambda|\left(\frac{1}{1-|\lambda|^{2}}\right)^{\frac{1}{2}}
$$

The case where $n \geq 2$. From now on, we write $\|\cdot\|_{2}$ for the $H^{2}$ norm $\|\cdot\|_{H^{2}}$. First, we prove the left-hand side inequality. Let

$$
e_{n}=\frac{\left(1-r^{2}\right)^{\frac{1}{2}}}{1-r z} b_{r}^{n-1} .
$$

Then $e_{n} \in K_{b_{r}^{n}}$ and $\left\|e_{n}\right\|_{2}=1$ (see [N1, Malmquist-Walsh Lemma, p. 116]). Moreover,

$$
\begin{aligned}
e_{n}^{\prime} & =\frac{r\left(1-r^{2}\right)^{\frac{1}{2}}}{(1-r z)^{2}} b_{r}^{n-1}+(n-1) \frac{\left(1-r^{2}\right)^{\frac{1}{2}}}{1-r z} b_{r}^{\prime} b_{r}^{n-2} \\
& =-\frac{r}{\left(1-r^{2}\right)^{\frac{1}{2}}} b_{r}^{\prime} b_{r}^{n-1}+(n-1) \frac{\left(1-r^{2}\right)^{\frac{1}{2}}}{1-r z} b_{r}^{\prime} b_{r}^{n-2},
\end{aligned}
$$

because $b_{r}^{\prime}=\frac{r^{2}-1}{(1-r z)^{2}}$. Then

$$
e_{n}^{\prime}=b_{r}^{\prime}\left[-\frac{r}{\left(1-r^{2}\right)^{\frac{1}{2}}} b_{r}^{n-1}+(n-1) \frac{\left(1-r^{2}\right)^{\frac{1}{2}}}{1-r z} b_{r}^{n-2}\right],
$$

and

$$
\begin{aligned}
\left\|e_{n}^{\prime}\right\|_{2}^{2}=\frac{1}{2 \pi} \int_{\mathbb{T}}\left|b_{r}^{\prime}(w)\right|\left|b_{r}^{\prime}(w)\right| \mid & -\frac{r}{\left(1-r^{2}\right)^{\frac{1}{2}}}\left(b_{r}(w)\right)^{n-1} \\
& +\left.(n-1) \frac{\left(1-r^{2}\right)^{\frac{1}{2}}}{1-r w}\left(b_{r}(w)\right)^{n-2}\right|^{2} d m(w) \\
=\frac{1}{2 \pi} \int_{\mathbb{T}}\left|b_{r}^{\prime}(w)\right|\left|b_{r}^{\prime}(w)\right| \mid & -\frac{r}{\left(1-r^{2}\right)^{\frac{1}{2}}} b_{r}(w)+\left.(n-1) \frac{\left(1-r^{2}\right)^{\frac{1}{2}}}{1-r w}\right|^{2} d m(w) .
\end{aligned}
$$

Using the variables $u=b_{r}(w)$, we obtain

$$
\left\|e_{n}^{\prime}\right\|_{2}^{2}=\frac{1}{2 \pi} \int_{\mathbb{T}}\left|b_{r}^{\prime}\left(b_{r}(u)\right)\right|-\frac{r}{\left(1-r^{2}\right)^{\frac{1}{2}}} u+\left.(n-1) \frac{\left(1-r^{2}\right)^{\frac{1}{2}}}{1-r b_{r}(u)}\right|^{2} d m(u) .
$$


But $1-r b_{r}=\frac{1-r z-r(r-z)}{1-r z}=\frac{1-r^{2}}{1-r z}$ and $b_{r}^{\prime} \circ b_{r}=\frac{r^{2}-1}{\left(1-r b_{r}\right)^{2}}=-\frac{\left(1-r z^{2}\right.}{1-r^{2}}$. This implies

$$
\begin{aligned}
\left\|e_{n}^{\prime}\right\|_{2}^{2} & =\frac{1}{2 \pi} \int_{\mathbb{T}}\left|\frac{(1-r u)^{2}}{1-r^{2}} \|-\frac{r}{\left(1-r^{2}\right)^{\frac{1}{2}}} u+(n-1) \frac{\left(1-r^{2}\right)^{\frac{1}{2}}}{1-r^{2}}(1-r u)\right|^{2} d m(u) \\
& =\frac{1}{\left(1-r^{2}\right)^{2}} \frac{1}{2 \pi} \int_{\mathbb{T}}|(1-r u)(-r u+(n-1)(1-r u))|^{2} d m(u) .
\end{aligned}
$$

Without loss of generality, we can replace $r$ by $-r$, which gives

$$
\left\|e_{n}^{\prime}\right\|_{2}=\frac{1}{\left(1-r^{2}\right)}\left\|\varphi_{n}\right\|_{2}
$$

where $\varphi_{n}=(1+r z)(r z+(n-1)(1+r z))$. Expanding, we get

$$
\begin{aligned}
\varphi_{n} & =(1+r z)(n r z+(n-1))=n r z+(n-1)+n r^{2} z^{2}+(n-1) r z \\
& =(n-1)+(n r+(n-1) r) z+n r^{2} z^{2},
\end{aligned}
$$

and

$$
\begin{aligned}
& \left\|e_{n}^{\prime}\right\|_{2}^{2}=\frac{1}{\left(1-r^{2}\right)^{2}}\left((n-1)^{2}+(2 n-1)^{2} r^{2}+n^{2} r^{4}\right) \\
& =\frac{n^{2}}{\left(1-r^{2}\right)^{2}}\left(1+4 r^{2}+r^{4}-\frac{2}{n}-\frac{4 r^{2}}{n}+\frac{1}{n^{2}}+\frac{r^{2}}{n^{2}}\right) \\
& =\left(\frac{n}{1-r}\right)^{2}\left(\frac{1}{1+r}\right)^{2}\left(1+4 r^{2}+r^{4}-\frac{2}{n}-\frac{4 r^{2}}{n}+\frac{1+r^{2}}{n^{2}}\right) \\
& =\left(\frac{n}{1-r}\right)^{2}\left(\frac{1}{1+r}\right)^{2}\left(1+4 r^{2}+r^{4}-5 r^{4}+5 r^{4}-\frac{4 r^{4}}{n}+\frac{4 r^{4}}{n}-\frac{4 r^{2}}{n}-\frac{2}{n}+\frac{1+r^{2}}{n^{2}}\right) \\
& =\left(\frac{n}{1-r}\right)^{2}\left(\frac{1}{1+r}\right)^{2}\left(4 r^{2}\left(1-r^{2}\right)-\frac{4 r^{2}}{n}\left(1-r^{2}\right)+\frac{1+r^{2}}{n^{2}}+1+5 r^{4}-\frac{4 r^{4}}{n}-\frac{2}{n}\right) \\
& =\left(\frac{n}{1-r}\right)^{2}\left(\frac{1}{1+r}\right)^{2}\left(4 r^{2}\left(1-r^{2}\right)\left(1-\frac{1}{n}\right)+\frac{1+r^{2}}{n^{2}}+1+5 r^{4}-\frac{4 r^{4}}{n}-\frac{2}{n}\right) \\
& \geq\left(\frac{n}{1-r}\right)^{2}\left(\frac{1}{1+r}\right)^{2}\left\{\begin{array}{l}
1+5 r^{4}-\frac{4 r^{4}}{n}-\frac{2}{n} \\
\frac{1}{4}+1+5 r^{4}-\frac{4 r^{4}}{2}-\frac{2}{2} \quad \text { if } n=2
\end{array}\right. \\
& \geq\left(\frac{n}{1-r}\right)^{2}\left(\frac{1}{1+r}\right)^{2}\left(1+5 r^{4}-\frac{4 r^{4}}{n}-\min \left(\frac{3}{4}, \frac{2}{n}\right)\right),
\end{aligned}
$$

and

$$
a(n, r) \geq \frac{1}{1+r}\left(1+5 r^{4}-\frac{4 r^{4}}{n}-\min \left(\frac{3}{4}, \frac{2}{n}\right)\right)^{\frac{1}{2}}
$$

which completes the proof of the left-hand side inequality. 
We pass to the right-hand side inequality. Let $\sigma$ be a sequence in $\mathbb{D}$ such that $1 \leq$ $\# \sigma \leq n,|\lambda| \leq r$ for all $\lambda \in \sigma$. Using [Z1, Proposition 4.1], we have

$$
\begin{aligned}
\|D\|_{K_{B_{\sigma}} \rightarrow H^{2}} & \leq \frac{1}{1-r}+\frac{1+r}{1-r}(n-1)+\frac{1}{1-r} \sqrt{n-2} \\
& =\frac{1}{1-r}(1+(1+r)(n-1)+\sqrt{n-2}) \\
& =\frac{1}{1-r}(n(1+r)-r+\sqrt{n-2})=\frac{n}{1-r}\left(1+r-\frac{r}{n}+\sqrt{\frac{1}{n}-\frac{2}{n^{2}}}\right) \\
& \leq \frac{n}{1-r}\left(1+r+\sqrt{\frac{1}{n}}\right)
\end{aligned}
$$

which gives the result.

Proof of (ii). Step 1. First, we prove the right-hand side inequality:

$$
\limsup _{n \rightarrow \infty} \frac{1}{n} C_{n, r} \leq \frac{1+r}{1-r}
$$

which becomes obvious because

$$
\|D\|_{K_{B_{\sigma}} \rightarrow H^{2}} \leq \frac{n}{1-r}\left(1+r+\sqrt{\frac{1}{n}}\right) .
$$

Step 2. Now we prove the left-hand side inequality:

$$
\liminf _{n \rightarrow \infty} \frac{1}{n} C_{n, r} \geq \frac{1+r}{1-r} .
$$

More precisely, we show that

$$
\liminf _{n \rightarrow \infty} \frac{1}{n}\|D\|_{K_{b_{r}^{n}} \rightarrow H^{2}} \geq \frac{1+r}{1-r} .
$$

Let $f \in K_{b_{r}^{n}}$. Then

$$
\begin{aligned}
f^{\prime} & =\left(f, e_{1}\right)_{H^{2}} \frac{r}{(1-r z)} e_{1}+\sum_{k=2}^{n}(k-1)\left(f, e_{k}\right)_{H^{2}} \frac{b_{r}^{\prime}}{b_{r}} e_{k}+r \sum_{k=2}^{n}\left(f, e_{k}\right)_{H^{2}} \frac{1}{(1-r z)} e_{k} \\
& =r \sum_{k=1}^{n}\left(f, e_{k}\right)_{H^{2}} \frac{1}{(1-r z)} e_{k}+\frac{1-r^{2}}{(1-r z)(z-r)} \sum_{k=2}^{n}(k-1)\left(f, e_{k}\right)_{H^{2}} e_{k} \\
& =\frac{r\left(1-r^{2}\right)^{\frac{1}{2}}}{(1-r z)^{2}} \sum_{k=1}^{n}\left(f, e_{k}\right)_{H^{2}} b_{r}^{k-1}+\frac{\left(1-r^{2}\right)^{\frac{3}{2}}}{(1-r z)^{2}(z-r)} \sum_{k=2}^{n}(k-1)\left(f, e_{k}\right)_{H^{2}} b_{r}^{k-1} \\
& =-b_{r}^{\prime}\left[\frac{r}{\left(1-r^{2}\right)^{\frac{1}{2}}} \sum_{k=1}^{n}\left(f, e_{k}\right)_{H^{2}} b_{r}^{k-1}+\frac{\left(1-r^{2}\right)^{\frac{1}{2}}}{z-r} \sum_{k=2}^{n}(k-1)\left(f, e_{k}\right)_{H^{2}} b_{r}^{k-1}\right] .
\end{aligned}
$$

Using the change of variables $v=b_{r}(u)$, we get

$$
\begin{aligned}
\left\|f^{\prime}\right\|_{2}^{2} & =\int_{\mathbb{T}}\left|b_{r}^{\prime}(u)\right|\left|b_{r}^{\prime}(u)\right|\left|\frac{r}{\left(1-r^{2}\right)^{\frac{1}{2}}} \sum_{k=1}^{n}\left(f, e_{k}\right)_{H^{2}} b_{r}^{k-1}+\frac{\left(1-r^{2}\right)^{\frac{1}{2}}}{u-r} \sum_{k=2}^{n}(k-1)\left(f, e_{k}\right)_{H^{2}} b_{r}^{k-1}\right|^{2} d u \\
& =\int_{\mathbb{T}}\left|b_{r}^{\prime}\left(b_{r}(v)\right)\right|\left|\frac{r}{\left(1-r^{2}\right)^{\frac{1}{2}}} \sum_{k=1}^{n}\left(f, e_{k}\right)_{H^{2}} v^{k-1}+\frac{\left(1-r^{2}\right)^{\frac{1}{2}}}{b_{r}(v)-r} \sum_{k=2}^{n}(k-1)\left(f, e_{k}\right)_{H^{2}} v^{k-1}\right|^{2} d v .
\end{aligned}
$$

But

$$
b_{r}-r=\frac{r-z-r(1-r z)}{1-r z}=\frac{z\left(r^{2}-1\right)}{1-r z}
$$


and

$$
b_{r}^{\prime} \circ b_{r}=\frac{r^{2}-1}{\left(1-r b_{r}\right)^{2}}=-\frac{(1-r z)^{2}}{1-r^{2}}
$$

which gives

$$
\begin{aligned}
&\left\|f^{\prime}\right\|_{H^{2}}^{2}=\frac{1}{1-r^{2}} \int_{\mathbb{T}}\left|(1-r v)^{2}\right| \mid \frac{r}{\left(1-r^{2}\right)^{\frac{1}{2}}} \sum_{k=1}^{n}\left(f, e_{k}\right)_{H^{2}} v^{k-1} \\
& \quad+\left.\frac{\left(1-r^{2}\right)^{\frac{1}{2}}}{v\left(r^{2}-1\right)}(1-r v) \sum_{k=2}^{n}(k-1)\left(f, e_{k}\right)_{H^{2}} v^{k-1}\right|^{2} d v \\
&=\frac{1}{\left(1-r^{2}\right)^{2}} \int_{\mathbb{T}}\left|(1-r v)^{2}\right|\left|r \sum_{k=1}^{n}\left(f, e_{k}\right)_{H^{2}} v^{k-1}-(1-r v) \sum_{k=2}^{n}(k-1)\left(f, e_{k}\right)_{H^{2}} v^{k-2}\right|^{2} d v \\
&=\frac{1}{\left(1-r^{2}\right)^{2}} \int_{\mathbb{T}}\left|r(1-r v) \sum_{k=0}^{n-1}\left(f, e_{k+1}\right)_{H^{2}} v^{k}-(1-r v)^{2} \sum_{k=0}^{n-2}(k+1)\left(f, e_{k+2}\right)_{H^{2}} v^{k}\right|^{2} d v
\end{aligned}
$$

But

$$
\left\|r(1-r v) \sum_{k=0}^{n-1}\left(f, e_{k+1}\right)_{H^{2}} v^{k}\right\|_{2} \leq r(1+r)\left(\sum_{k=0}^{n-1}\left|\left(f, e_{k+1}\right)_{H^{2}}\right|^{2}\right)^{1 / 2} \leq r(1+r)\|f\|_{2},
$$

and in particular, if $\|f\|_{2}=o(n)$ as $n \rightarrow \infty$, then

$$
\lim _{n \rightarrow \infty} \frac{1}{n}\left\|r(1-r v) \sum_{k=0}^{n-1}\left(f, e_{k+1}\right)_{H^{2}} v^{k}\right\|_{2}=0 .
$$

Now,

$$
\begin{aligned}
(1-r v)^{2} \sum_{k=0}^{n-2}(k+1)\left(f, e_{k+2}\right)_{H^{2}} v^{k}=\left(1-2 r v+r^{2} v^{2}\right) \sum_{k=0}^{n-2}(k+1)\left(f, e_{k+2}\right)_{H^{2}} v^{k} \\
=\sum_{k=0}^{n-2}(k+1)\left(f, e_{k+2}\right)_{H^{2}} v^{k}-2 r \sum_{k=0}^{n-2}(k+1)\left(f, e_{k+2}\right)_{H^{2}} v^{k+1} \\
\quad+r^{2} \sum_{k=0}^{n-2}(k+1)\left(f, e_{k+2}\right)_{H^{2}} v^{k+2} \\
\quad \sum_{k=0}^{n-2}(k+1)\left(f, e_{k+2}\right)_{H^{2}} v^{k}-2 r \sum_{k=1}^{n-1} k\left(f, e_{k+1}\right)_{H^{2}} v^{k}+r^{2} \sum_{k=2}^{n}(k-1)\left(f, e_{k}\right)_{H^{2}} v^{k} \\
=\left(f, e_{2}\right)_{H^{2}}+2\left(f, e_{3}\right)_{H^{2}} v \\
\quad+\sum_{k=2}^{n-2}\left[(k+1)\left(f, e_{k+2}\right)_{H^{2}}-2 r k\left(f, e_{k+1}\right)_{H^{2}}+r^{2}(k-1)\left(f, e_{k}\right)_{H^{2}}\right] v^{k} \\
\quad-2 r\left[\left(f, e_{2}\right)_{H^{2}} v+(n-1)\left(f, e_{n}\right)_{H^{2}} v^{n-1}\right] \\
\quad+r^{2}\left[(n-2)\left(f, e_{n-1}\right)_{H^{2}} v^{n-1}+(n-1)\left(f, e_{n}\right)_{H^{2}} v^{n}\right] \\
=\left(f, e_{2}\right)_{H^{2}}+2\left[\left(f, e_{3}\right)_{H^{2}}-r\left(f, e_{2}\right)_{H^{2}}\right] v \\
\quad+\sum_{k=2}^{n-2}\left[(k+1)\left(f, e_{k+2}\right)_{H^{2}}-2 r k\left(f, e_{k+1}\right)_{H^{2}}+r^{2}(k-1)\left(f, e_{k}\right)_{H^{2}}\right] v^{k} \\
\quad+\left[r^{2}(n-2)\left(f, e_{n-1}\right)_{H^{2}}-2 r(n-1)\left(f, e_{n}\right)_{H^{2}}\right] v^{n-1}+r^{2}(n-1)\left(f, e_{n}\right)_{H^{2}} v^{n} .
\end{aligned}
$$


Since

$$
\begin{aligned}
& \frac{1}{n} \frac{1}{\left(1-r^{2}\right)}\left[\left\|(1-r v)^{2} \sum_{k=0}^{n-2}(k+1)\left(f, e_{k+2}\right)_{H^{2}} v^{k}\right\|_{2}+\left\|r(1-r v) \sum_{k=0}^{n-1}\left(f, e_{k+1}\right)_{H^{2}} v^{k}\right\|_{2}\right] \\
& \quad \geq \frac{1}{n}\left\|f^{\prime}\right\|_{H^{2}} \\
& \quad \geq \frac{1}{n} \frac{1}{\left(1-r^{2}\right)}\left[\left\|(1-r v)^{2} \sum_{k=0}^{n-2}(k+1)\left(f, e_{k+2}\right)_{H^{2}} v^{k}\right\|_{2}-\left\|r(1-r v) \sum_{k=0}^{n-1}\left(f, e_{k+1}\right)_{H^{2}} v^{k}\right\|_{2}\right],
\end{aligned}
$$

we see that, if $\|f\|_{2}=o(n)$ as $n \rightarrow \infty$, then

$$
\begin{gathered}
\frac{1}{1+r} \liminf _{n \rightarrow \infty} \frac{1}{n\|f\|_{2}}\left\|(1-r v)^{2} \sum_{k=0}^{n-2}(k+1)\left(f, e_{k+2}\right)_{H^{2}} v^{k}\right\|_{2} \geq \liminf _{n \rightarrow \infty} \frac{1-r}{n} \frac{\left\|f^{\prime}\right\|_{2}}{\|f\|_{2}} \\
\geq \frac{1}{1+r} \liminf _{n \rightarrow \infty} \frac{1}{n\|f\|_{2}}\left\|(1-r v)^{2} \sum_{k=0}^{n-2}(k+1)\left(f, e_{k+2}\right)_{H^{2}} v^{k}\right\|_{2}
\end{gathered}
$$

This gives

$$
\liminf _{n \rightarrow \infty} \frac{1-r}{n} \frac{\left\|f^{\prime}\right\|_{2}}{\|f\|_{2}}=\frac{1}{1+r} \liminf _{n \rightarrow \infty} \frac{1}{n\|f\|_{2}}\left\|(1-r v)^{2} \sum_{k=0}^{n-2}(k+1)\left(f, e_{k+2}\right)_{H^{2}} v^{k}\right\|_{2} .
$$

But

$$
\begin{aligned}
\|(1-r v)^{2} & \sum_{k=0}^{n-2}(k+1)\left(f, e_{k+2}\right)_{H^{2}} v^{k} \|_{2}^{2} \\
=\left|\left(f, e_{2}\right)_{H^{2}}\right|^{2}+4\left|\left(f, e_{3}\right)_{H^{2}}-r\left(f, e_{2}\right)_{H^{2}}\right|^{2} & \\
& +\left|r^{2}(n-2)\left(f, e_{n-1}\right)_{H^{2}}-2 r(n-1)\left(f, e_{n}\right)_{H^{2}}\right|^{2}+r^{4}(n-1)^{2}\left|\left(f, e_{n}\right)_{H^{2}}\right|^{2} \\
& +\sum_{k=2}^{n-2}\left|(k+1)\left(f, e_{k+2}\right)_{H^{2}}-2 r k\left(f, e_{k+1}\right)_{H^{2}}+r^{2}(k-1)\left(f, e_{k}\right)_{H^{2}}\right|^{2} .
\end{aligned}
$$

Now, let $s=s_{n}$ be a sequence of even integers such that

$$
\lim _{n \rightarrow \infty} s_{n}=\infty \quad \text { and } \quad s_{n}=o(n) \quad \text { as } \quad n \rightarrow \infty
$$

Consider the following function $f$ in $K_{b_{r}^{n}}$ :

$$
\begin{aligned}
f & =e_{n}-e_{n-1}+e_{n-2}-e_{n-3}+\cdots+(-1)^{k} e_{n-k}+\cdots+e_{n-s}-e_{n-s-1}+e_{n-s-2} \\
& =\sum_{k=0}^{s+2}(-1)^{k} e_{n-k} .
\end{aligned}
$$

With this $f$, we get

$$
\begin{aligned}
& \left\|(1-r v)^{2} \sum_{k=0}^{n-2}(k+1)\left(f, e_{k+2}\right)_{H^{2}} v^{k}\right\|_{2}^{2}=\left|r^{2}(n-2)+2 r(n-1)\right|^{2}+r^{4}(n-1)^{2} \\
& \quad+\sum_{l=2}^{n-2}\left|(n-l+1)\left(f, e_{n-l+2}\right)_{H^{2}}-2 r(n-l)\left(f, e_{n-l+1}\right)_{H^{2}}+r^{2}(n-l-1)\left(f, e_{n-l}\right)_{H^{2}}\right|^{2}
\end{aligned}
$$


with $l=n-k$ in the last sum. Finally, this gives

$$
\begin{aligned}
\left\|(1-r v)^{2} \sum_{k=0}^{n-2}(k+1)\left(f, e_{k+2}\right)_{H^{2}} v^{k}\right\|_{2}^{2} & =\left|r^{2}(n-2)+2 r(n-1)\right|^{2}+r^{4}(n-1)^{2} \\
& +\sum_{l=2}^{s+1}\left|(n-l+1)+2 r(n-l)+r^{2}(n-l-1)\right|^{2} \\
& +|(n-s-1)+2 r(n-s-2)|^{2}+|n-s-2|^{2} .
\end{aligned}
$$

Thus,

$$
\begin{aligned}
\left\|(1-r v)^{2} \sum_{k=0}^{n-2}(k+1)\left(f, e_{k+2}\right)_{H^{2}} v^{k}\right\|_{2}^{2} \geq & \left|r^{2}(n-2)+2 r(n-1)\right|^{2}+r^{4}(n-1)^{2} \\
& +s\left|(n-s)+2 r(n-s-1)+r^{2}(n-s-2)\right|^{2} \\
& +|(n-s-1)+2 r(n-s-2)|^{2}+|n-s-2|^{2} .
\end{aligned}
$$

In particular,

$$
\left\|(1-r v)^{2} \sum_{k=0}^{n-2}(k+1)\left(f, e_{k+2}\right)_{H^{2}} v^{k}\right\|_{2}^{2} \geq s\left|(n-s)+2 r(n-s-1)+r^{2}(n-s-2)\right|^{2} .
$$

Now, since

$$
\|f\|_{2}^{2}=s+3=s_{n}+3
$$

we get

$$
\begin{aligned}
& \liminf _{n \rightarrow \infty} \frac{1}{n^{2}\|f\|_{2}^{2}}\left\|(1-r v)^{2} \sum_{k=0}^{n-2}(k+1)\left(f, e_{k+2}\right)_{H^{2}} v^{k}\right\|_{2}^{2} \\
& \quad \geq \liminf _{n \rightarrow \infty} \frac{1}{n^{2}\|f\|_{2}^{2}}\left(\|f\|_{2}^{2}-3\right)\left|(n-s)+2 r(n-s-1)+r^{2}(n-s-2)\right|^{2} .
\end{aligned}
$$

Since

we obtain

$$
\lim _{n \rightarrow \infty} \frac{3}{n^{2} s_{n}^{2}}\left|(n-s)+2 r(n-s-1)+r^{2}(n-s-2)\right|^{2}=0,
$$

$$
\begin{aligned}
& \liminf _{n \rightarrow \infty} \frac{1}{n^{2}\|f\|_{2}^{2}}\left\|(1-r v)^{2} \sum_{k=0}^{n-2}(k+1)\left(f, e_{k+2}\right)_{H^{2}} v^{k}\right\|_{2}^{2} \\
& \quad \geq \liminf _{n \rightarrow \infty} \frac{1}{n^{2} s_{n}^{2}} s_{n}^{2}\left|\left(n-s_{n}\right)+2 r\left(n-s_{n}-1\right)+r^{2}\left(n-s_{n}-2\right)\right|^{2} \\
& \quad=\lim _{n \rightarrow \infty} \frac{1}{n^{2}}\left|\left(n-s_{n}\right)+2 r\left(n-s_{n}-1\right)+r^{2}\left(n-s_{n}-2\right)\right|^{2} \\
& \quad=\lim _{n \rightarrow \infty} \frac{1}{n^{2}}\left|n+2 r n+r^{2} n\right|^{2}=(1+r)^{4} .
\end{aligned}
$$

Now we can conclude that

$$
\begin{aligned}
& \liminf _{n \rightarrow \infty} \frac{1-r}{n}\|D\|_{K_{b_{r}^{n}} \rightarrow H^{2}} \geq \liminf _{n \rightarrow \infty} \frac{1-r}{n} \frac{\left\|f^{\prime}\right\|_{2}}{\|f\|_{2}} \\
& \quad=\frac{1}{1+r} \liminf _{n \rightarrow \infty} \frac{1}{n\|f\|_{2}}\left\|(1-r v)^{2} \sum_{k=0}^{n-2}(k+1)\left(f, e_{k+2}\right)_{H^{2}} v^{k}\right\|_{2} \\
& \quad \geq \frac{(1+r)^{2}}{1+r}=1+r .
\end{aligned}
$$


Step 3. Conclusion. Using Step 1 and Step 2, we get

$$
\limsup _{n \rightarrow \infty} \frac{1-r}{n} C_{n, r}=\liminf _{n \rightarrow \infty} \frac{1-r}{n} C_{n, r}=1+r,
$$

which means that the sequence $\left(\frac{1}{n} C_{n, r}\right)_{n \geq 1}$ is convergent and

$$
\lim _{n \rightarrow \infty} \frac{1}{n} C_{n, r}=\frac{1+r}{1-r}
$$

Comments. (a) Using [BoEr, Theorem 7.1.7, p. 324] (or equivalently, Levin's inequality [L]) and complex interpolation, this Bernstein-type inequality can be extended to $H^{p}$ spaces, $2 \leq p \leq \infty$. Of course, this was already known via Dyakonov's result Dya1, but our method could give a better numerical constants $c_{p}$ in the inequality

$$
\left\|f^{\prime}\right\|_{H^{p}} \leq c_{p}\left\|B^{\prime}\right\|_{\infty}\|f\|_{H^{p}} .
$$

The case where $1 \leq p \leq 2$ can be treated by using a result of Baranov ( $p=1$, private communication) and still complex interpolation.

(b) In the same spirit, it is also possible to generalize the above Bernstein-type inequality to the same class of rational functions $f$ in $\mathbb{D}$, replacing the Hardy space $H^{2}$ by the Besov spaces $B_{2,2}^{s}, s \in \mathbb{R}$, of all holomorphic functions $f=\sum_{k \geq 0} \widehat{f}(k) z^{k}$ in $\mathbb{D}$ satisfying

$$
\|f\|_{B_{2,2}^{s}}:=\left(\sum_{k \geq 0}(k+1)^{2 s}|\widehat{f}(k)|^{2}\right)^{\frac{1}{2}}<\infty .
$$

The same spaces are also known as Sobolev, or Hardy weighted spaces, or Dirichlet weighted spaces. (In particular, the classical Bergman space corresponds to $s=-\frac{1}{2}$ and the classical Dirichlet space corresponds to $s=\frac{1}{2}$.) Using the above approach, one can prove the sharpness of the growth order $\frac{n}{1-r}$ in the corresponding Bernstein-type inequality

$$
\left\|f^{\prime}\right\|_{B_{2,2}^{s}} \leq c_{s} \frac{n}{1-r}\|f\|_{B_{2,2}^{s}}
$$

(at least for integral values of $s$ ).

(c) One can also prove the inequality

$$
\|f\|_{B_{2,2}^{s}} \leq c_{s}^{\prime}\left(\frac{n}{1-r}\right)^{s}\|f\|_{H^{2}}
$$

for $s \geq 0$ and for the same class of functions (essentially, this inequality can be found in Dya2), and show the sharpness of the growth order $\left(\frac{n}{1-r}\right)^{s}$ (at least for integers $s$ ). An interesting application of this inequality lies in constrained Nevanlinna-Pick interpolation in weighted Hardy and Bergman spaces, see [Z1] and [Z2] for the details.

Notice that Dyn'kin (for example in Dyn]), and Pekarskiı̌ (in [Pe1, $[\mathrm{Pe} 2$ ] and [PeSt]) studied Bernstein-type inequalities for rational functions in Besov and Sobolev spaces. In particular, they applied such inequalities to inverse theorems of rational approximation. Our approach is different and more constructive. We are able to obtain uniform bounds depending on the geometry of poles of order $n$, which allows us to obtain estimates that are asymptotically sharp.

(d) The above comments can lead to the question what happens if we replace Besov spaces $B_{2,2}^{s}$ by other Banach spaces, for example by $W$, the Wiener algebra of absolutely convergent Taylor series. In this case, we obtain

$$
\|f\|_{W} \leq c(n, r)\|f\|_{H^{2}}
$$


where $c(n, r) \leq c\left(\frac{n^{2}}{1-r}\right)^{\frac{1}{2}}$ and $c$ is a numerical constant. We suspect that $\left(\frac{n^{2}}{1-r}\right)^{\frac{1}{2}}$ is the right growth order of $c(n, r)$. An interesting application of this inequality to estimating the norm of the resolvent of a power-bounded matrix $T$ of size $m \times n$ on a Banach space was given in [Z3. Inequality (5) above is linked deeply with the inequality

$$
\left\|f^{\prime}\right\|_{H^{1}} \leq \gamma n\|f\|_{H^{\infty}}
$$

through Hardy's inequality:

$$
\|f\|_{W} \leq \pi\left\|f^{\prime}\right\|_{H^{1}}+|f(0)|,
$$

for all $f \in W$ (see N. Nikolski [N2, p. 370, 8.7.4-(c)]).

Inequality (6) was (shown and) used by LeVeque and Trefethen in [LeTr] with $\gamma=2$, and later by Spijker in Sp with $\gamma=1$ (an improvement) so as to apply it to the Kreiss Matrix Theorem, which asserts the uniform equivalence over all $(n \times n)$ matrices of power boundedness and a certain resolvent estimate. This allowed them to show that the ratio of the constants in these two conditions grows linearly with $n$.

\section{ACKNOWLEDGMENT}

I would like to thank Professor Nikolaĭ Nikolskiı for his invaluable help and his precious advices. I also would like to thank Professor Alexander Borichev for many helpful discussions.

\section{REFERENCES}

[B1] A. Baranov, Inégalités de Bernstein dans les espaces modèles et applications, Thèse soutenue à l'Université Bordeaux 1, 2005.

[B2] - Bernstein-type inequalities for shift-coinvariant subspaces and their applications to Carleson embeddings, J. Funct. Anal. 223 (2005), no. 1, 116-146. MR2139883 (2007b:46041)

[B3] - Embeddings of model subspaces of the Hardy class: compactness and Schatten-von Neumann ideals, Izv. Ross. Akad. Nauk. Ser. Mat. 73 (2009), no. 6, 3-28; English transl., Izv. Math. 73 (2009), no. 6, 1077-1100. MR2640976

[BoEr] P. Borwein and T. Erdélyi, Polynomials and polynomial inequalities, Grad. Texts in Math., vol. 161, Springer-Verlag, New York, 1995. MR1367960 (97e:41001)

[DeLo] R. A. DeVore and G. G. Lorentz, Constructive approximation, Grundlehren Math. Wiss., Bd. 303, Springer-Verlag, Berlin, 1993. MR1261635 (95f:41001)

[Dya1] K. Dyakonov, Differentiation in star-invariant subspaces. I. Boundedness and compactness, J. Funct. Anal. 192 (2002), 364-386. MR.1923406 (2003g:30060)

[Dya2] _ Smooth functions in the range of a Hankel operator, Indiana Univ. Math. J. 43 (1994), 805-838. MR1923406 (2003g:30060)

[Dyn] E. M. Dyn'kin, Inequalities for rational functions, J. Approx. Theory 91 (1997), no. 3, 349-367. MR1486473 (99a:41010)

[LeTr] R. J. LeVeque and L. N. Trefethen, On the resolvent condition in the Kreiss matrix theorem, BIT 24 (1984), 584-591. MR0764830 (86c:39004)

[L] M. B. Levin, Estimation of the derivative of a meromorphic function on the boundary of the domains, Teor. Funktsiü Funktsional. Anal. i Prilozhen., vyp. 24, Vishcha Shkola, Kharkov, 1975, pp. 68-85. (Russian) MR0402053 (53:5876)

[N1] N. K. Nikol'skiu, Lectures on the shift operators, Nauka, Moscow, 1980; English transl., Treatise on the shift operator. Spectral function theory, Grundlehren Math. Wiss., Bd. 273, SpringerVerlag, Berlin, 1986, MR.0575166 (82i:47013) MR0827223 (87i:47042)

[N2] - Operators, functions, and systems: an easy reading. Vol. 1, Math. Surveys Monogr., vol. 92, Amer. Math. Soc., Providence, RI, 2002. MR 1864396 (2003i:47001a)

[Pe1] A. A. Pekarskiı̌, Inequalities of Bernstein type for derivatives of rational functions, and inverse theorems of rational approximation, Mat. Sb. (N. S.) 124 (1984), no. 4, 571-588; English transl., Math. USSR-Sb. 52 (1985), 557-574. MR0754478(86a:41017)

[Pe2] - Estimates of the higher derivatives of rational functions and their applications, Izv. Akad. Nauk BSSR Ser. Fiz.-Mat. Nauk 1980, no. 5, 21-28. (Russian) MR0599155 (82a:41016) 
[PeSt] A. A. Pekarskiŭ and G. Shtal', Bernstein-type inequalities for derivatives of rational functions in $L_{p}$ spaces, $p<1$, Mat. Sb. 186 (1995), no. 6, 119-130; English transl., Sb. Math. 186 (1995), no. 1, 121-131. MR.1641684 (99f:41014)

[RaSc] Q. I. Rahman and G. Schmeisser, Analytic theory of polynomials, London Math. Soc. Monogr. New Ser., vol. 26, Clarendon Press, Oxford, 2002. MR.1954841 (2004b:30015)

[Sp] M. N. Spijker, On a conjecture by LeVeque and Trefethen related to the Kreiss matrix theorem, BIT 31 (1991), 551-555. MR1127492 (92h:15012)

[Z1] R. Zarouf, Effective $H^{\infty}$ interpolation constrained by Hardy and Bergman norms (submitted).

[Z2] _ Effective $H^{\infty}$ interpolation constrained by Hardy and Bergman weighted norms (submitted).

[Z3] Une amélioration d'un résultat de E. B. Davies et B. Simon, C. R. Math. Acad. Sci. Paris 347 (2009), no. 15-16, 939-942. MR2542898(2010i:47042)

CMi-LATP, UMR 6632, Université de Provence, 39 rue F.-Joliot-Curie, 13453 Marseille CEDEX 13, FRANCE

E-mail address: rzarouf@cmi.univ-mrs.fr

Received 29/JAN/2010

Originally published in English 